\title{
Spacetimes foliated by Killing horizons
}

\author{
Tomasz Pawlowski ${ }^{1,3}$, Jerzy Lewandowski ${ }^{1,3,4}$ and Jacek Jezierski ${ }^{2,3}$ \\ ${ }^{1}$ Instytut Fizyki Teoretycznej, Uniwersytet Warszawski, ul. Hoża 69, 00-681 Warsaw, Poland \\ 2 Katedra Metod Matematycznych Fizyki, Uniwersytet Warszawski, ul. Hoża 74, \\ 00-682 Warsaw, Poland \\ ${ }^{3}$ Max Planck Institut für Gravitationsphysik, Albert Einstein Institut, 14476 Golm, Germany \\ ${ }^{4}$ Physics Department, 104 Davey, Penn State, University Park, PA 16802, USA
}

Received 16 October 2003

Published 27 January 2004

Online at stacks.iop.org/CQG/21/1237 (DOI: 10.1088/0264-9381/21/4/033)

\begin{abstract}
It seems to be expected that a horizon of a quasi-local type, such as a Killing or an isolated horizon, by analogy with a globally defined event horizon, should be unique in some open neighbourhood in the spacetime, provided the vacuum Einstein or the Einstein-Maxwell equations are satisfied. The aim of our paper is to verify whether that intuition is correct. If one can extend a so-called Kundt metric, in such a way that its null, shear-free surfaces have spherical spacetime sections, the resulting spacetime is foliated by socalled non-expanding horizons. The obstacle is Kundt's constraint induced at the surfaces by the Einstein or the Einstein-Maxwell equations, and the requirement that a solution be globally defined on the sphere. We derived a transformation (reflection) that creates a solution to Kundt's constraint out of data defining an extremal isolated horizon. Using that transformation, we derived a class of exact solutions to the Einstein or Einstein-Maxwell equations of very special properties. Each spacetime we construct is foliated by a family of the Killing horizons. Moreover, it admits another, transversal Killing horizon. The intrinsic and extrinsic geometries of the transversal Killing horizon coincide with the one defined on the event horizon of the extremal Kerr-Newman solution. However, the Killing horizon in our example admits yet another Killing vector tangent to and null at it. The geometries of the leaves are given by the reflection.
\end{abstract}

PACS numbers: $04.20 . \mathrm{Ex}, 04.70 . \mathrm{Bw}, 11.10 . \mathrm{Ef}$

\section{Introduction}

In the standard black-hole theory an event horizon is defined as a boundary of a certain distinguished region of spacetime. On the other hand, quasi-local definitions of horizons are 
known which lead to a local black-hole physics and geometry [1-10]. Typically, a horizon is a cylinder formed by 2-surfaces diffeomorphic with the 2-sphere. In the stationary black-hole case, the cylinder is expected to be a null surface. If the intrinsic geometry of the surface is preserved by a tangent null flow, then the cylinder is called a non-expanding, shear-free horizon. If both the intrinsic and extrinsic geometries are preserved by a tangent null flow, then the cylinder becomes an isolated horizon (see $[5,6]$ for details). In a very special case, there is a Killing vector field defined in a neighbourhood of the cylinder and tangent to its null generators. Then we deal with a Killing ${ }^{5}$ horizon. It seems to be expected that a horizon of this quasi-local type, by analogy with the globally defined event horizon, should be unique in some open neighbourhood in the given vacuum spacetime. The aim of our paper is to verify whether that intuition is correct. We would like to prove or disprove the existence of a solution of vacuum Einstein's or the Einstein-Maxwell equations foliated by non-expanding, shear-free horizons. A metric tensor of this type necessarily belongs to Kundt's class ${ }^{6}$ [12]. The structure of Einstein's and the Einstein-Maxwell equations in this class is discussed in [12] and a large family of exact solutions is known. The new issue is the quasi-global assumption that null, non-expanding, shear-free surfaces foliating a given Kundt solution have spacelike spherical sections.

We approach this problem in terms of the non-expanding horizons geometry. Vacuum Einstein's equations imposed on a metric tensor which admits a foliation by non-expanding, shear-free horizons induce on each horizon certain constraint equations on the intrinsic geometry and the rotation 1-form potential. We construct a transformation that maps the constraints into another set of equations satisfied by the intrinsic geometry and the rotation 1 -form potential of an extremal isolated horizon contained in a vacuum spacetime. The inverse transform applied to the intrinsic geometry and the rotation 1-form potential defined on the extremal Kerr event horizon provides a solution to our constraints! The vacuum constraints and the transformation are generalized to the Einstein-Maxwell case. Whereas the nature of that transformation is somewhat mysterious in the general case considered here, it becomes clear in the case of the examples we derive later. Next, we extend appropriately every solution to the vacuum (electrovac) constraint equations into a solution to the vacuum Einstein (Einstein-Maxwell) equations. The derived class of solutions admits a two-dimensional Lie algebra of the Killing vector fields generated by $K_{0}, K_{1}$ which satisfy $\left[K_{0}, K_{1}\right]=K_{0}$. For every value of $u_{0}$, the vector field $u_{0} K_{0}-K_{1}$ defines a bifurcated Killing horizon (that is it is tangent to a pair of Killing horizons which share a spherical slice). All the bifurcated Killing horizons share a single Killing horizon tangent to $K_{0}$. In other words, the resulting spacetime is foliated by the Killing horizons, and admits one more Killing horizon transversal to the leaves of the foliation. The transversal Killing horizon equipped with the Killing vector $K_{0}$ is an extremal vacuum (electrovac) isolated horizon. Its intrinsic geometry, the rotation 1-form potential and the electromagnetic field are related to the data defined on each leaf of the foliation exactly by the transformation discussed above. Finally, we derive an explicit form of the solution corresponding to the extremal Kerr-Newman horizons.

The constraint equations considered as equations of certain data defined on a manifold diffeomorphic with a 2-sphere are interesting by themselves. According to the results of [13], the only axi-symmetric solutions are those given by the Kerr-Newman extremal horizon and the transform. It is not known if there are any other solutions. Some partial results are included in the appendix.

5 We follow the definition of a Killing horizon presented in [11].

6 The class of metric such that there exists null non-expanding non-twisting congruence. 
In conclusion, our results contradict even the belief that a Killing horizon should be unique in some spacetime neighbourhood. However our examples are quasi-local and their geodesic extension should be understood.

\section{Non-expanding horizons}

We recall in this section the definition of the non-expanding horizons and properties relevant in this paper [6]. A non-expanding horizon is defined to be a null 3-surface $\triangle$ contained in a four-dimensional spacetime of the Lorentz signature, such that

(i) $\triangle$ is an embedding of $S \times \mathcal{I}$, where the manifolds $S$ and $\mathcal{I}$ are, respectively, the 2-sphere and the one-dimensional interval,

(ii) for every $s \in S$, the embedding maps $\{s\} \times \mathcal{I}$ into a null curve,

(iii) every null vector field tangent to $\triangle$ is non-expanding.

It follows from the Raychaudhuri equation that if the stress energy tensor satisfies at every point of $\triangle$ the non-negativity condition

$$
T_{\mu \nu} \ell^{\mu} \ell^{\nu} \geqslant 0
$$

where $\ell$ is a null vector tangent to $\triangle$, then $\triangle$ is also shear free. It means that the pullback $q$ of the spacetime metric $g$ onto $\triangle$ is transversal to $\ell$ and Lie dragged by $\ell$,

$$
\ell\lrcorner q=0, \quad \mathcal{L}_{\ell} q=0 .
$$

It follows that the spacetime covariant derivative $\nabla$ reduces naturally to a covariant derivative $\mathbb{D}$ defined in the tangent bundle $T \triangle$, that is for every two vector fields $X, Y$ tangent to $\triangle$, so is $\nabla_{X} Y$. It also follows that the null direction tangent to $\triangle$ is covariantly constant, hence for every null vector field $\ell$ tangent to $\triangle$,

$$
\mathbb{D} \ell=\omega \otimes \ell
$$

where $\omega$ is a differential 1 -form defined on $\triangle$, called the rotation 1-form potential. We can always choose $\ell$ to be geodesic, that is such that $\nabla_{\ell} \ell=0$. Then necessarily [6]

$$
\ell\lrcorner \omega=0, \quad \mathcal{L}_{\ell} \omega=0 .
$$

Let us fix an orientation and a time orientation in $M$. An orientation in $S$ is adjusted in the following way: let $\ell$ be a future-oriented null vector tangent to $\Delta$ at $x$, and $n$ be another future-oriented null vector at $x$. A frame $(X, Y)$ tangent to $S$ at $p(x)$, where $p: \Delta \rightarrow S$ is the natural projection, has a positive orientation whenever the orientation of the frame $(X, Y, n, \ell)$ is positive.

If the non-expanding horizon is contained in an Einstein-Maxwell vacuum, then the electromagnetic field tensor $F$ defines on $\triangle$ yet another transversal to (and Lie dragged by) $\ell$ object, namely the pullback $\mathcal{F}$ of the self-dual part $\frac{1}{2}(F-\mathrm{i} \star F)$ of $F$ onto $\triangle$,

$$
\left.\left.\ell\lrcorner \mathcal{F}=0, \quad \mathcal{L}_{\ell} \mathcal{F}=\ell\right\lrcorner \mathrm{d} \mathcal{F}+\mathrm{d}(\ell\lrcorner \mathcal{F}\right)=0 .
$$

The first equality is a consequence of the Raychaudhuri equation, which implies [15] that (orthogonal to $\ell$ ) 1-form $\ell\lrcorner(F-\mathrm{i} \star F)$ is null on the horizon. Therefore, it must be proportional to $\ell_{a}$. The second equality follows from Maxwell equations.

It follows from (2), (4), (5) that, there are defined on $S$ : a metric tensor $\hat{q}$, a 1-form $\hat{\omega}$ and a complex-valued differential 2 -form $\hat{\mathcal{F}}$, such that

$$
q=p^{*} \hat{q}, \quad \omega=p^{*} \hat{\omega}, \quad \mathcal{F}=p^{*} \hat{\mathcal{F}} .
$$

We call $(\hat{q}, \hat{\omega}, \hat{\mathcal{F}})$, respectively, a projected 2 -metric tensor, a projected rotation 1 -form potential and a projected electromagnetic field 2 -form induced on $S$ by $(\triangle, \ell)$. The 2 -form $\hat{\mathcal{F}}$ 
can be represented by a projective complex electromagnetic scalar $\Phi_{1}$, respectively defined on S by

$$
\hat{\mathcal{F}}=: \mathrm{i} \Phi_{1} \hat{\epsilon},
$$

where $\hat{\epsilon}$ is the volume 2-form on $S$ defined by the metric tensor $\hat{q}$ and the orientation.

Note that $\ell$ given as above can always be replaced by $\ell^{\prime}=f \ell$, where $f$ is a positive function such that $\ell^{\mu} f_{, \mu}=0$. The metric tensor $\hat{q}$ and the complex-valued differential 2-form $\mathcal{F}$ defined on $S$ are independent of that choice, whereas $\omega^{\prime}=\omega+\mathrm{d} \ln f$. We will see below, however, that in the case of a one-dimensional family of non-expanding horizons foliating a spacetime, that freedom will be restricted to $f=$ const.

In the example of spacetime found in this paper, we encounter a pair of non-expanding horizons which share a sphere. If this is the case, the structures induced on $S$ are related to each other. Indeed, suppose $\triangle^{\prime}$ is another non-expanding horizon in $M$ such that $\triangle \cap \triangle^{\prime}=\tilde{S}$, where $\tilde{S}$ is diffeomorphic to a sphere. Let $\ell^{\prime}$ be a null vector field tangent to $\triangle^{\prime}$ such that

$$
\left.\ell^{\mu} \ell_{\mu}^{\prime}\right|_{\tilde{S}}=-1 \text {. }
$$

The spheres of the null geodesics $S$ and $S^{\prime}$ can be naturally identified. Then, the corresponding projective metric tensors $\hat{q}$ and $\hat{q}^{\prime}$ and the projective electromagnetic field 2-forms $\hat{\mathcal{F}}, \hat{\mathcal{F}}^{\prime}$ just coincide because they are defined by the pullback to the single sphere $\tilde{S}$ of, respectively, the metric tensor and the self-dual electromagnetic field $(F-\mathrm{i} \star F)$. The orientations of $S$ corresponding to $\triangle$ and $\triangle^{\prime}$ are opposite to each other; therefore, projective complex electromagnetic scalars $\Phi_{1}, \Phi_{1}^{\prime}$ are also opposite. The pullbacks $\omega_{(\tilde{S})}$ and $\omega_{(\tilde{S})}^{\prime}$ of the rotation 1-form potentials $\omega$ and $\omega^{\prime}$ to $\tilde{S}$ are related in the following way:

$$
\omega_{(\tilde{S})}^{\prime}=\left(\ell_{\mu} \nabla \ell^{\prime \mu}\right)_{(\tilde{S})}=-\left(\ell_{\mu}^{\prime} \nabla \ell^{\mu}\right)_{(\tilde{S})}=-\omega_{(\tilde{S})} .
$$

In summary, we have

$\hat{q}^{\prime}=\hat{q}, \quad \hat{\omega}^{\prime}=-\hat{\omega}, \quad \Phi_{1}^{\prime}=-\Phi_{1}, \quad$ orientation $^{\prime}=-$ orientation.

\section{Foliations by non-expanding horizons}

We consider in this work a spacetime $(M, g)$ foliated by non-expanding horizons. Our considerations are quasi-local, in the sense that we assume that

$$
M=S \times \mathcal{I} \times \mathcal{I},
$$

where $S$ is a manifold diffeomorphic with the 2-sphere, and $\mathcal{I}$ is an interval,

(ii) for every $u^{\prime} \in \mathcal{I}$, the 3 -surface $S \times \mathcal{I} \times\left\{u^{\prime}\right\}$ is a non-expanding horizon,

(iii) every curve $\{s\} \times \mathcal{I} \times\left\{u^{\prime}\right\}$ such that $s \in S$ and $u^{\prime} \in \mathcal{I}$ is null.

Every spacetime foliated by the non-expanding horizons can be introduced (quasi-locally) in this way. Let $u$ be a real-valued function defined on $M$ such that for every $u^{\prime} \in \mathcal{I}, u$ is constant on the corresponding non-expanding horizon $S \times \mathcal{I} \times\left\{u^{\prime}\right\}$ and

$$
\mathrm{d} u \neq 0
$$

for every $x \in M$. A function $u$ of those properties can be given by any coordinate defined in $R$. For every value $u_{0}$ of the function $u$, the non-expanding horizon $S \times \mathcal{I} \times\left\{u^{\prime}\right\}$ on which $u$ takes that value henceforth will be denoted by $\triangle_{u_{0}}$ (often we will drop the suffix 0 at $u$ ). The function $u$ provides in $M$ a null vector field $\ell$,

$$
\ell^{\mu}:=-g^{\mu v} u_{, v}
$$


geodesic,

$$
\nabla_{\ell} \ell=0
$$

and tangent at every $x \in M$ to $\triangle_{u(x)}$. Therefore, for every non-expanding horizon $\triangle_{u}$ the results of the previous section apply to the vector field $\ell$ defined in (13). In conclusion, for every leaf $\triangle_{u}$ of the foliation, $S$ is equipped with the metric tensor $\hat{q}$, the projected rotation 1 -form potential $\hat{\omega}$ and the projected self-dual electromagnetic field $\hat{\mathcal{F}}$ as was explained in the previous section. The structure $(\hat{q}, \hat{\omega}, \hat{\mathcal{F}})$ arbitrarily depends on the leaf $\triangle_{u}$.

There is still some freedom in the definition of the vector field $\ell$ on $M$. The function $u$ can be replaced by $\tilde{u}=h(u)$, where $h$ is an arbitrary function whose gradient nowhere vanishes. That transformation amounts to the rescaling of $\ell$ by a factor $h^{\prime}(u)$ constant on every leaf $\triangle_{u}$. The transformation leaves $(\hat{q}, \hat{\omega}, \hat{\mathcal{F}})$ invariant. Therefore, the structure $(\hat{q}, \hat{\omega}, \hat{\mathcal{F}})$ is uniquely defined on $S$ and depends only on leaf $\triangle_{u}$ and the spacetime metric tensor $g$.

\section{Constraints}

Imposing the Einstein-Maxwell equations on the spacetime foliated by non-expanding horizons implies interesting constraints on the structures $(\hat{q}, \hat{\omega}, \mathcal{F})$ defined on $S$. We formulate and discuss the constraints in this section. The derivation will be presented in section 6 in which we analyse the full set of Einstein-Maxwell equations imposed on a spacetime foliated by non-expanding horizons. Let us begin with the Einstein vacuum case, that is $F=0$ in $M$.

Proposition 1. Suppose $(M, g)$ is a four-dimensional spacetime foliated by non-expanding horizons in the meaning of the conditions (i)-(iii) of section 3; suppose g satisfies the vacuum Einstein equations. Then, the following constraint is satisfied on the manifold $S$ for every leaf $\triangle_{u}$,

$$
\hat{D}_{B} \hat{\omega}_{A}+\hat{D}_{A} \hat{\omega}_{B}-2 \hat{\omega}_{A} \hat{\omega}_{B}+\hat{R}_{A B}=0,
$$

where we denote by $\hat{D}$ and $\hat{R}$ the covariant derivative and the Ricci tensor, respectively, of the 2-metric tensor $\hat{q}$ defined on $S$.

In the presence of the electromagnetic field $F$, a constraint implied by the Maxwell equations will be added (and the first constraint will be modified by the non-zero $F$ ). It is convenient to express the Maxwell constraint by the complex structure on $S$ compatible with the orientation of $S$ distinguished in section 2 . Let $(z, \bar{z})$ be a local complex coordinate system in $S$ such that the orientation of the coframe $(\operatorname{Re} \mathrm{d} z, \operatorname{Im~d} z)$ is consistent with the orientation of $S$. We decompose every differential 1 -form $k=k_{z} \mathrm{~d} z+k_{\bar{z}} \mathrm{~d} \bar{z}$ defined on $S$ in the following way,

$$
k^{(1,0)}=k_{z} \mathrm{~d} z, \quad k^{(0,1)}=k_{\bar{z}} \mathrm{~d} \bar{z},
$$

and in particular for every complex-valued function $f$ defined on $S$ we define

$$
\partial f=\mathrm{d} f^{(1,0)}, \quad \bar{\partial} f=\mathrm{d} f^{(0,1)} .
$$

Proposition 2. Suppose $(M, g)$ is a four-dimensional spacetime foliated by non-expanding horizons in the meaning the conditions (i)-(iii) of section 3; suppose F is an electromagnetic vector field in $M$ such that $(g, F)$ satisfy the vacuum Einstein-Maxwell equations. Then, the following constraints are satisfied on the manifold $S$ for every leaf $\triangle_{u}$,

$$
\begin{aligned}
& \hat{D}_{B} \hat{\omega}_{A}+\hat{D}_{A} \hat{\omega}_{B}-2 \hat{\omega}_{A} \omega_{B}+\hat{R}_{A B}-2 \kappa_{0}\left|\Phi_{1}\right|^{2} \hat{q}_{A B}=0, \\
& \left(\partial-2 \hat{\omega}^{(1,0)}\right) \Phi_{1}=0 .
\end{aligned}
$$


The rotation 1-form $\hat{\omega}$ can be decomposed according to the Hodge decomposition [14] on the sphere in the following way:

$$
\hat{\omega}=\hat{*} \mathrm{~d} U+\mathrm{d} \ln B,
$$

where $U, B$ are real functions. Then constraint $(18 b)$ can be easily integrated giving the following form of $\Phi_{1}$ as a function of $(U, B)$,

$$
\Phi_{1}=E_{0} B^{2} \mathrm{e}^{2 \mathrm{i} U}, \quad E_{0}=\text { const. }
$$

This equation was investigated in more detail in [13].

Obviously proposition 1 is a special case of proposition 2 . The proof of proposition 2 follows in a straightforward way from the Einstein-Maxwell equations discussed in section 6.

The constraints considerably restrict possible 2-metric tensors $\hat{q}$, the rotation 1-form potential $\hat{\omega}$ and electromagnetic field induced on $S$. Their strength consists in the fact that solutions have to be defined globally on $S$.

On the other hand, we will also show in section 7 that for every single solution of the constrains (18) there exists an electrovac $(M, g, F)$ foliated by non-expanding horizons.

\section{A transform providing solutions to the constraints}

There is a remarkable mathematical relation between the constraints (15) and the constraints satisfied by the geometry of a vacuum extremal-isolated horizon [13]. The relation generalizes to the constraints (18) and the constraints satisfied by the geometry and the electromagnetic field on an extremal electrovacuum isolated horizon [13]. (By 'mathematical', we mean that the constraints are not the same, but there is a transformation that maps solutions of one set of the constraints into another.) Let us recall that an extremal isolated horizon $\left(\triangle^{\prime}, \ell^{\prime}\right)$ in a non-expanding horizon $\triangle^{\prime}$ equipped with a tangent null vector field such that

$$
\left[\mathcal{L}_{\ell^{\prime}}, \mathbb{D}^{\prime}\right]=0, \quad \text { and } \quad \mathbb{D}_{\ell^{\prime}}^{\prime} \ell^{\prime}=0 .
$$

At $\triangle^{\prime}$, the vacuum Einstein equations induce the following constraint equations on the metric tensor $\hat{q}^{\prime}$ and the rotation 1-form potential $\hat{\omega}^{\prime}$ projected onto the manifold $S$ [13] (we are still using the notation of section 2)

$$
\hat{D}_{B}^{\prime} \hat{\omega}_{A}^{\prime}+\hat{D}_{A}^{\prime} \hat{\omega}_{B}^{\prime}+2 \hat{\omega}_{A}^{\prime} \hat{\omega}_{B}^{\prime}-\hat{R}_{A B}^{\prime}=0 .
$$

Comparing the equations above with the vacuum constraints (15) one can easily see the relation:

Theorem 1. The following map

$$
\hat{\omega}^{\prime} \mapsto \hat{\omega}=-\hat{\omega}^{\prime}, \quad \hat{q}^{\prime} \mapsto \hat{q}=\hat{q}^{\prime}
$$

is a bijection of the set of solutions to the vacuum extremal-isolated horizon constraints (22) onto the set of solutions to the constraints (15). In particular, the projected metric tensor $\hat{q}^{\prime}$ and the projected rotation 1-form potential $\hat{\omega}^{\prime}$ induced on the event horizon in the extremal Kerr spacetime are mapped by the transformation (23) into a solution to the constraints (15).

In the presence of the electromagnetic field $F^{\prime}$ at an extremal isolated horizon $\triangle^{\prime}$, the Einstein-Maxwell equations and an assumption that the electromagnetic field is Lie dragged by the vector field $\ell^{\prime}$ (see [13] for the details) amount to the following constraint equations:

$$
\begin{aligned}
& \hat{D}_{B}^{\prime} \hat{\omega}_{A}^{\prime}+\hat{D}_{A}^{\prime} \hat{\omega}_{B}^{\prime}+2 \hat{\omega}_{A}^{\prime} \hat{\omega}_{B}^{\prime}-\hat{R}_{A B}^{\prime}+2 \kappa_{0}\left|\Phi_{1}^{\prime}\right|^{2} \hat{q}_{A B}=0, \\
& \left(\bar{\partial}+2 \hat{\omega}^{(0,1)}\right) \Phi_{1}^{\prime}=0 .
\end{aligned}
$$


A comparison of the equations above with the electrovac constraints (18) shows that theorem 1 generalizes to the following:

Theorem 2. The map (23) accompanied by

$$
\Phi_{1}^{\prime} \mapsto \Phi_{1}=\overline{\Phi_{1}^{\prime}}
$$

is a bijection of the set of solutions to the extremal electrovac-isolated horizon constraints (24) onto the set of solutions to the constraints (18). In particular, the projected metric tensor $\hat{q}^{\prime}$, the projected rotation 1-form potential $\hat{\omega}^{\prime}$ and the projected electromagnetic field $\hat{\mathcal{F}}^{\prime}$ induced on the event horizon in the extremal Kerr-Newman spacetime are mapped by the transformation (23), (25) into a solution to the constraints (18).

The relevance of theorems 1 and 2 above consists in establishing the existence of nontrivial, globally defined on $S$ solutions to the vacuum constraints (15) and, respectively, the electrovac constraints (18).

Remark 1. Applied to the constraints (18), the transformation (23), (25) is equivalent (up to change of orientation $)^{7}$ to the transformation (10) mapping into each other data corresponding to two intersecting non-expanding horizons. That observation may be considered as an indication of the possible existence of a non-expanding horizon transversal to one of the horizons $\triangle_{u}$. This is exactly what happens in the case of the class of solutions constructed in the next section.

\section{Proof of propositions 1,2}

Every spacetime $(M, g)$ foliated by non-expanding horizons in the sense of the conditions (i)-(iii) of section 3 can be represented by $M=S \times \mathcal{I} \times \mathcal{I}$ and the following metric tensor (see section 31.2 in [12] for a general form of spacetime foliated by null, non-expanding, shear-free surfaces):

$$
g=\hat{q}-2 \mathrm{~d} u(\mathrm{~d} v+\hat{W}+H \mathrm{~d} u)
$$

where

(a) the function $v$ (respectively, $u$ ) is a parametrization of the first (second) factor $\mathcal{I}$ of the product (11) extended naturally to $S \times \mathcal{I} \times \mathcal{I}$,

(b) $\hat{q}$ is a metric tensor defined on $S$ and depending on the value of $u$, naturally lifted to the product $S \times \mathcal{I} \times \mathcal{I}$,

(c) $\hat{W}(H)$ is a differential 1-form (a function) defined on $S$ depending on the values of $u$ and $v$ and extended naturally to the product $S \times \mathcal{I} \times \mathcal{I}$.

Let $z, \bar{z}$ be any (local) coordinate system defined on $S$ and extended naturally to some domain in $S \times \mathcal{I} \times \mathcal{I}$. In terms of the coordinate system $(z, \bar{z}, v, u)$, equation (26) reads

$$
g=2 P^{-2} \mathrm{~d} z \mathrm{~d} \bar{z}-2 \mathrm{~d} u(\mathrm{~d} v+W \mathrm{~d} z+\bar{W} \mathrm{~d} \bar{z}+H \mathrm{~d} u) \quad P_{, v}=0,
$$

where $P$ is a real valued and $W$ is a complex-valued function. The constancy surfaces of $u$ are non-expanding horizons.

The converse is also true: given a metric tensor (27), a surface $\Delta_{u_{0}}=S \times \mathcal{I} \times\left\{u_{0}\right\}$ is a non-expanding horizon for every value of $u_{0}$ taken by the functions $v, u$, respectively.

7 Transformation (10) changes the orientation of the 2D slice $\tilde{S}$ preserving spacetime orientation. That transformation modified to preserve the orientation of the slice changes the spacetime orientation (affecting the Hodge dual in the definition of $\mathcal{F}$ ). That modification implies the transformation of $\Phi_{1}$ to be of the form given by (25). 
We express now all the structures defined in sections 2, 3 by the components of the metric tensor above. To begin with, the function $u$ is the same as in section 3 , and the complex-valued function $z$ is the same as $z$ in section 4 . In this coordinate system the vector field $\ell(13)$ is

$$
\ell^{\mu} \partial_{\mu}=\partial_{v} .
$$

The structures $\hat{q}$ and $\hat{\omega}$ introduced in $S$ for every value taken by the function $u$ are

$$
\hat{q}=2 P^{-2} \mathrm{~d} z \mathrm{~d} \bar{z}, \quad \hat{\omega}=\frac{1}{2}\left(W_{, v} \mathrm{~d} z+\bar{W}_{, v} \mathrm{~d} \bar{z}\right) .
$$

Note that equation (4) is equivalent to

$$
W_{, v v}=0 .
$$

Every electromagnetic field $F$ defined on $M$ which satisfies the conditions (5) can be written as

$F=\Phi_{1}\left(e^{4} \wedge e^{3}+e^{2} \wedge e^{1}\right)+\Phi_{2} e^{2} \wedge e^{3}+\overline{\Phi_{1}}\left(e^{4} \wedge e^{3}-e^{2} \wedge e^{1}\right)+\overline{\Phi_{2}} e^{1} \wedge e^{3} \quad \Phi_{1, v}=0$,

where $\left(e^{1}, e^{2}, e^{3}, e^{4}\right)$ is the coframe dual to the following null frame

$$
e_{1}=\bar{e}_{2}=P \partial_{z}, \quad e_{3}=\partial_{u}+P^{2}\left(\bar{W} \partial_{z}+W \partial_{\bar{z}}\right)-\left(H+P^{2} W \bar{W}\right) \partial_{v}, \quad e_{4}=\partial_{v} .
$$

Then, $\Phi_{1}$ is the same function as the one introduced in section 2 and the complex-valued differential 2-form $\hat{\mathcal{F}}$ defined in the same section on $S$ is

$$
\hat{\mathcal{F}}=-\frac{\Phi_{1}}{P^{2}} \mathrm{~d} \bar{z} \wedge \mathrm{d} z
$$

In the construction we have already taken into account a part of the Einstein-Maxwell equations. Therefore, our metric tensor $g$ (27), (30) and the electromagnetic field $F$ (31) already satisfy

$$
\begin{aligned}
& R_{44}=\kappa_{0} T_{44}=0=R_{41}=\kappa_{0} T_{41}=R_{42}=\kappa_{0} T_{42}, \\
& \left.\left.\left.\left.e_{1}\right\lrcorner e_{4}\right\lrcorner \mathrm{~d}(F-\mathrm{i} * F)=0=e_{2}\right\lrcorner e_{4}\right\lrcorner \mathrm{d}(F-\mathrm{i} * F) .
\end{aligned}
$$

Every metric tensor given by (27), (30) belongs to Kundt's class. We apply below the discussion of the structure of the Einstein-Maxwell equations for this class which can be found in [12].

We turn now to the proof of propositions 1 and 2. Consider the following Einstein equations

$\left(P^{2} W_{, v}\right)_{, z}-\frac{1}{2} P^{2}\left(W_{, v}\right)^{2}=R_{11}=\kappa_{0} T_{11}=0$,

$2 P^{2}(\ln (P))_{, z \bar{z}}+\frac{1}{2} P^{2}\left(\bar{W}_{, v z}+W_{, v \bar{z}}-W_{, v} \bar{W}_{, v}\right)=R_{12}=\kappa_{0} T_{12}=2 \kappa_{0} \Phi_{1} \overline{\Phi_{1}}$,

where in each of the lines above, the first and the third equality is an identity. Using the relations between $P$ and the 2-metric tensor $\hat{q}$ and between $W_{, v}$ and $\hat{\omega}$ (see (29)), one easily recognizes equation $(18 a)$ and, in the vacuum case, equation (15).

Finally, one of the Maxwell equations reads

$$
\left.\left.\left.e_{3}\right\lrcorner e_{4}\right\lrcorner e_{1}\right\lrcorner \mathrm{d}(F-\mathrm{i} \star F)=\frac{1}{P}\left(\Phi_{1, z}-W_{, v} \Phi_{1}\right)=0,
$$

where the first equality is an identity. This is exactly equation $(18 b)$. This completes the proof. 


\section{A class of solutions to the Einstein-Maxwell equations}

Let us assume in this section that we are given $W_{, v}(z, \bar{z}, u), P(z, \bar{z}, u)$ and $\Phi_{1}(z, \bar{z}, u)$ such that the constraints $(18 a),(18 b)$ are satisfied for every sphere $u=$ const, $v=$ const. We turn now to the issue of the existence of solutions to the full set of the Einstein-Maxwell equations.

The second derivative with respect to $v$ of the function $H$ is given by the $(\mu, \nu)=(3,4)$ component of the Einstein-Maxwell equations, namely

$H_{, v v}=2 \kappa_{0}\left|\Phi_{1}\right|^{2}+\operatorname{div} \hat{\omega}-2 \hat{q}^{A B} \hat{\omega}_{A} \hat{\omega}_{B}=2 \kappa_{0}\left|\Phi_{1}\right|^{2}+\frac{1}{2} P^{2}\left(\bar{W}_{, v z}+W_{, v \bar{z}}-2 W_{, v} \bar{W}_{, v}\right)$,

where $\operatorname{div} \hat{\omega}:=\hat{q}^{A B} \hat{D}_{A} \hat{\omega}_{B}$ and $\hat{q}^{A B}$ is the two-dimensional inverse metric.

Similarly, the $v$ derivative of the remaining component $\Phi_{2}$ is involved in $\left.\left.\left.e_{3}\right\lrcorner e_{2}\right\lrcorner e_{4}\right\lrcorner \mathrm{d}(F-$ $\mathrm{i} \star F)$ and the corresponding Maxwell equation gives

$$
\Phi_{2, v}=P\left(\Phi_{1, \bar{z}}+\bar{W}_{, v} \Phi_{1}\right) .
$$

In particular, it follows from (37), (38) that $H_{, v v v}=0$ and $\Phi_{2, v v}=0$. Hence, the most general form of the functions $W, H, \Phi_{2}$ is ${ }^{8}$

$$
W(z, \bar{z}, u, v)=W_{, v}(z, \bar{z}, u) v+W_{0}(z, \bar{z}, u),
$$

$H(z, \bar{z}, u, v)=\left[\kappa_{0}\left|\Phi_{1}\right|^{2}+\frac{1}{4} P^{2}\left(\bar{W}_{, v z}+W_{, v \bar{z}}-2 W_{, v} \bar{W}_{, v}\right)\right] v^{2}+G_{0}(z, \bar{z}, u) v+H_{0}(z, \bar{z}, u)$,

$\Phi_{2}(z, \bar{z}, u, v)=P\left(\Phi_{1, \bar{z}}+\bar{W}_{, v} \Phi_{1}\right) v+\Phi_{2}^{0}(z, \bar{z}, u)$.

The remaining Einstein-Maxwell equations amount to the following system of equations on $W_{0}, G_{0}, H_{0}, P_{, u}, W_{, v u}$ [12] (the first two equations are explicitly written down in [12], but to propose a solution we also needed the last equation below, therefore the first two were included for completeness):

$$
\begin{aligned}
2 \kappa_{0} \Phi_{1} \Phi_{2}^{0}= & P\left(P^{2} W^{0}\right)_{, z \bar{z}}+P\left[(\ln (P))_{, u}-\frac{1}{2} P^{2} \bar{W}_{, z}^{0}-\frac{1}{2} P^{2} W_{\bar{z}}^{0}\right]_{, z} \\
& +\frac{1}{2} P\left[\left(P^{2} W_{, v}\right)_{, z} \bar{W}^{0}+\left(P^{2} \bar{W}_{, v}\right)_{z} W^{0}\right]-2 P_{, \bar{z}}\left(P^{2} W^{0}\right)_{, z} \\
& +P G_{, z}^{0}+\frac{1}{2} P^{3}\left[\left(W^{0} \bar{W}_{, v}\right)_{, z}-\left(W^{0} W_{, v}\right)_{, \bar{z}}\right]+P_{, u}-\frac{1}{2} P W_{, v u}, \\
P \Phi_{2, z}^{0}=P_{, z} \Phi_{2}^{0} & +\Phi_{1, u}-2(\ln (P))_{, u} \Phi_{1}+P^{2}\left[\left(\bar{W}^{0} \Phi_{1}\right)_{, z}+\left(W^{0} \Phi_{1}\right)_{, \bar{z}}\right], \\
2 \kappa_{0} \Phi_{2}^{0} \bar{\Phi}_{2}^{0}=2 & P^{2} H_{, z \bar{z}}^{0}+P^{2}\left[\left(\bar{W}_{, v} H^{0}\right)_{, z}+\left(W_{, v} H^{0}\right)_{, \bar{z}}\right]+2 P^{2} s_{, z \bar{z}}^{0}+P^{2}\left[\left(\bar{W}_{, v} s^{0}\right)_{, z}\right. \\
& \left.+\left(W_{, v} s^{0}\right)_{, \bar{z}}\right]-2\left(P^{2} \bar{W}^{0}\right)_{, \bar{z}}\left(P^{2} W^{0}\right)_{, z}-2\left[P^{2}\left(\mu_{, z}^{0} \bar{W}^{0}+\mu_{, \bar{z}}^{0} W^{0}\right)\right. \\
& \left.+\mu_{, u}^{0}+\mu^{0}\left(G^{0}+P^{2}\left(W^{0} \bar{W}_{, v}+\bar{W}^{0} W_{, v}\right)+\mu^{0}\right)\right],
\end{aligned}
$$

where

$$
s^{0}=P^{2} W^{0} \bar{W}^{0} \quad \mu^{0}=\frac{1}{2} P^{2}\left(\bar{W}_{, z}^{0}+W_{, \bar{z}}^{0}\right)-(\ln (P))_{, u} .
$$

It is easy to see that the following example defines a solution of (40)

$$
P_{, u}=W_{, v u}=\Phi_{1, u}=0=W^{0}=G^{0}=H^{0}=\Phi_{2}^{0} .
$$

Therefore, the following has been shown:

Theorem 3. For every 2-metric tensor $\hat{q}$, a differential 1 -form $\hat{\omega}$ and a complex-valued function $\Phi_{1}$ all defined globally on $S$ and solving the constraint equations (18a), (18b), the metric tensor

8 For the components of the decomposition we follow the notation of section 31.4 of [12]. 
$g$ and electromagnetic field $F$ defined by (42) are a solution of the Einstein-Maxwell equations. If $\Phi_{1}=0$, then all the resulting electromagnetic field $F=0$.

Corollary 1. Combining theorem 3 with theorems 1 and 2, we established the existence of solutions to vacuum Einstein's equations foliated by non-expanding horizons, and solutions to the vacuum Einstein-Maxwell equations foliated by non-expanding horizons.

We discuss now the structure of the spacetime given by theorem 3 and by a solution $\left(\hat{q}, \hat{\omega}, \Phi_{1}\right)$ of the constraint equations $(18 a),(18 b)$.

The metric tensor $g$ has two Killing vector fields, namely

$$
K_{0}=\partial_{u} \quad \text { and } \quad K_{1}=u \partial_{u}-v \partial_{v} .
$$

For every value of $u_{0}$, the corresponding leaf $\triangle_{u_{0}}$ is tangent to the Killing vector field $K_{1}-u_{0} K_{0}$. Moreover, $K_{1}-u_{0} K_{0}$ is null on $\triangle_{u_{0}}$. Therefore, all the leaves are the Killing horizons. Every $\left(\triangle_{u_{0}}, K_{1}-u_{0} K_{0}\right)$ is non-extremal in the sense that on $\triangle_{u_{0}}$

$$
\nabla_{\left(K_{1}-u_{0} K_{0}\right)}\left(K_{1}-u_{0} K_{0}\right)=-\left(K_{1}-u_{0} K_{0}\right) \neq 0 .
$$

The Killing vector $K_{0}$ is transversal to every leaf $\Delta_{u_{0}}$. The one-dimensional group of isometries it generates maps one leaf into another. The spacetime pseudo-norm of $K_{0}$ equals

$$
g_{u u}=-2 H=-\left[2 \kappa_{0}\left|\Phi_{1}\right|^{2}+\frac{1}{2} P^{2}\left(\bar{W}_{, v z}+W_{, v \bar{z}}-2 W_{, v} \bar{W}_{, v}\right)\right] v^{2} .
$$

Therefore, $K_{0}$ becomes null on the surface $v=0$. Let us denote that surface by $\triangle^{o}$. The surface $\triangle^{o}$ is null itself and tangent to $K_{0}$. Hence $\triangle^{o}$ is yet another Killing horizon. It has a certain quite special property: there are two distinct null Killing vectors tangent to $\triangle^{o}$. Indeed, the other one is the vector field $K_{1}$. On $\triangle^{o}$ they satisfy

$$
\nabla_{K_{0}} K_{0}=0, \quad \nabla_{K_{1}} K_{1}=K_{1} .
$$

Therefore $\left(\triangle^{o}, K_{0}\right)$ is extremal whereas $\left(\triangle^{o}, K_{1}\right)$ is non-extremal.

Consider finally the projected metric, projected rotation 1-form potential and the projected electromagnetic field defined on the sphere $S$ by $\left(\triangle^{o}, K_{0}\right)$ (see section 2). Denote them by $\hat{q}^{o}, \hat{\omega}^{o}$ and $\hat{\mathcal{F}}^{o}$, respectively. Because the horizons $\triangle^{o}$ and $\triangle_{u_{0}}$ share a sphere, and

$$
g\left(K_{0}, \ell\right)=g_{u v}=-1,
$$

the structure $\left(\hat{q}^{o}, \hat{\omega}^{o}, \hat{\mathcal{F}}^{o}\right)$ is related to the structure $(\hat{q}, \hat{\omega}, \hat{\mathcal{F}})$ (see remark 1 )

$$
\hat{q}^{o}=\hat{q}, \quad \hat{\omega}^{o}=-\hat{\omega}, \quad \Phi_{1}^{o}=\bar{\Phi}_{1} .
$$

This is exactly the transformation (23), (25).

\section{The axi-symmetric example}

In the previous section, we constructed a class of solutions to the Einstein-Maxwell equations labelled by solutions to the constraints (18). We complete now the task of the construction of an explicit electrovac example by deriving the axi-symmetric solutions to the constraints ${ }^{9}$.

Let $(\theta, \varphi)$ be a spherical coordinate system on $S$. We consider now the constraint equations $(18)$ in the case the data $(\hat{q}, \hat{\omega}, \hat{\mathcal{F}})$ are invariant with respect to the group of

9 The general solution $\left(\hat{q}, \hat{\omega}, \Phi_{1}\right)$ of the constraint equations (18) is not known. See [13] and the appendix for a discussion. The general axi-symmetric solution was derived in [13]. It is given via theorem 2 by the Kerr-Newman solution. Since the equation (131a) in [13] contains a misprint made in the process of rewriting the results, we outline the derivation in this section. 
diffeomorphisms of $S$ generated by the vector field $\frac{\partial}{\partial \varphi}$. We replace the coordinate function $\theta$ by a function $x$ naturally defined by the area (2-volume) form $\hat{\epsilon}$ corresponding to the metric tensor $\hat{q}$, and by the symmetry generator $\boldsymbol{\Phi}$, namely $x$ such that

$$
\mathrm{d} x=\Phi\lrcorner \hat{\epsilon}, \quad x \in\left[-\frac{A}{4 \pi}, \frac{A}{4 \pi}\right], \quad A=\int_{S} \hat{\epsilon} .
$$

The complex coordinate $z$ used in the previous section is related to the new coordinates $(x, \varphi)$ in the following way:

$$
\mathrm{d} z=\frac{1}{\sqrt{2}}\left(P^{2}(x) \mathrm{d} x+\mathrm{i} \mathrm{d} \varphi\right)
$$

so $x$ is equivalent to coordinate $z$ defined in (31.41) of [12].

The condition that the 2-metric tensor $\hat{q}$ (see (29)) is of the class $C^{1}$ on $S$ implies the following conditions on the function $P$ to be satisfied at the poles:

$$
\lim _{x \rightarrow \pm \frac{A}{4 \pi}} \frac{1}{P}=0 \quad \lim _{x \rightarrow \pm \frac{A}{4 \pi}}\left(\frac{1}{P^{2}}\right)_{, x}=\mp 2 .
$$

After applying this coordinate system and equation (20), the constraint (18a) (with $\hat{\omega}$ decomposed by (19)) takes the following form:

$$
\begin{aligned}
& \left(\frac{1}{P B}\right)_{, x x}+\frac{\left(P_{, x}\right)^{2}}{P^{3} B}+\frac{U_{x}^{2}}{P B}+2 \kappa_{0}\left|E_{0}\right|^{2} P B^{3}=0, \\
& \left(\frac{1}{B}\right)_{, x x}-\frac{\left(U_{, x}\right)^{2}}{B}=0, \\
& \left(\frac{U_{, x}}{B^{2}}\right)_{, x}=0
\end{aligned}
$$

where $(52 a)$ is the trace of $(18 a)$, and $(52 b),(52 c)$ are the result of splitting the traceless part of $(18 a)$ into the real and, respectively, imaginary part.

The set of solutions to the equations (52) and to the globality conditions (51) can be labelled by three real parameters $\alpha, A, \theta_{0}$ such that

$$
\alpha \in[0,1], \quad A \in] 0, \infty\left[, \quad \theta_{0} \in[0,2 \pi[.\right.
$$

And the general solution is

$$
\begin{aligned}
& P^{2}(x)=\frac{2 \pi\left(1+\alpha^{2}\right)}{A} \frac{A^{2}+\frac{1-\alpha^{2}}{1+\alpha^{2}}(4 \pi x)^{2}}{A^{2}-(4 \pi x)^{2}}, \\
& U(x)= \pm \arctan \left(4 \pi \sqrt{\frac{1-\alpha^{2}}{1+\alpha^{2}}} \frac{x}{A}\right), \\
& B(x)=\left(1+\frac{1-\alpha^{2}}{1+\alpha^{2}} \frac{(4 \pi x)^{2}}{A^{2}}\right)^{-\frac{1}{2}}, \\
& \Phi_{1}(x)=\mathrm{e}^{\mathrm{i} \theta_{0}} \sqrt{\frac{2 \pi}{\kappa_{0}}} \frac{2 \alpha A^{\frac{3}{2}}}{1+\alpha^{2}} \frac{\left(A^{2}-\frac{1-\alpha^{2}}{1+\alpha^{2}}(4 \pi x)^{2}\right) \pm 2 \mathrm{i} A \sqrt{\frac{1-\alpha^{2}}{1+\alpha^{2}}}(4 \pi x)}{\left(A^{2}+\frac{1-\alpha^{2}}{1+\alpha^{2}}(4 \pi x)^{2}\right)^{2}} .
\end{aligned}
$$

It is easy to show [13] that this solution corresponds via the transformation $P \rightarrow P, \omega \rightarrow-\omega$, $\left.\Phi_{1} \rightarrow \bar{\Phi}_{1}\right)$ to the extremal Kerr-Newman event horizon. The case $\alpha=0,1$, in particular, 
corresponds to the extremal Kerr and the extremal Reissner-Nordström event horizons, respectively.

In conclusion, the class of explicit examples of electrovacua foliated by the Killing horizons is given by the metric tensor (27) and the electromagnetic field (31) such that

$P^{2}=\frac{2 \pi\left(1+\alpha^{2}\right)}{A} \frac{A^{2}+\frac{1-\alpha^{2}}{1+\alpha^{2}}(4 \pi x)^{2}}{A^{2}-(4 \pi x)^{2}}$,

$W=\frac{2 \sqrt{2}\left(1-\alpha^{2}\right)^{\frac{1}{2}} A}{\left(1+\alpha^{2}\right)^{\frac{3}{2}}} \frac{A^{2}-(4 \pi x)^{2}}{\left(A^{2}+\frac{1-\alpha^{2}}{1+\alpha^{2}}(4 \pi x)^{2}\right)^{2}}\left( \pm \mathrm{i} A-\sqrt{\frac{1-\alpha^{2}}{1+\alpha^{2}}}(4 \pi x)\right) v$,

$H=4 \pi A \frac{\left(A^{2}+\frac{1-\alpha^{2}}{1+\alpha^{2}}(4 \pi x)^{2}\right)^{2}-4 \frac{1-\alpha^{2}}{1+\alpha^{2}} A^{2}\left(A^{2}-(4 \pi x)^{2}\right)}{\left(1+\alpha^{2}\right)\left(A^{2}+\frac{1-\alpha^{2}}{1+\alpha^{2}}(4 \pi x)^{2}\right)^{3}} v^{2}$,

$\Phi_{1}=\mathrm{e}^{\mathrm{i} \theta_{0}} \sqrt{\frac{2 \pi}{\kappa_{0}}} \frac{2 \alpha A^{\frac{3}{2}}}{1+\alpha^{2}} \frac{\left(A^{2}-\frac{1-\alpha^{2}}{1+\alpha^{2}}(4 \pi x)^{2}\right) \pm 2 \mathrm{i} A \sqrt{\frac{1-\alpha^{2}}{1+\alpha^{2}}}(4 \pi x)}{\left(A^{2}+\frac{1-\alpha^{2}}{1+\alpha^{2}}(4 \pi x)^{2}\right)^{2}}$,

$\Phi_{2}=\mathrm{e}^{\mathrm{i} \theta_{0}} \sqrt{\frac{2}{\kappa_{0}}} \frac{\alpha\left(1-\alpha^{2}\right)(8 \pi A)^{2} x}{\left(1+\alpha^{2}\right)^{\frac{5}{2}}} \frac{\left(A^{2}-(4 \pi x)^{2}\right)^{\frac{1}{2}}}{\left(A^{2}+\frac{1-\alpha^{2}}{1+\alpha^{2}}(4 \pi x)^{2}\right)^{\frac{7}{2}}}\left(A \pm \mathrm{i} \sqrt{\frac{1-\alpha^{2}}{1+\alpha^{2}}}(4 \pi x)\right)^{2} v$,

where $\mathrm{d} z$ is given for known $P(x)$ by (50).

In particular, the vacuum solutions are the type $D$ Kundt's solutions expressed in [12] by

$$
\begin{aligned}
& P^{2}=\frac{x^{2}+l^{2}}{k\left(\left(x^{2}-l^{2}\right)+2 m x\right)}, \\
& W=-\frac{\sqrt{2} v}{P^{2}(x-\mathrm{i} l)}, \\
& H=-\left(\frac{k}{2\left(x^{2}+l^{2}\right)}+\frac{2 l^{2}}{P^{2}\left(x^{2}+l^{2}\right)^{2}}\right) v^{2},
\end{aligned}
$$

where in our case the parameters $k, l, m$ are real and such that

$$
m=0, \quad-k=2 l=\frac{A}{2 \pi} .
$$

Comparing our results with [12] we may also conclude that the vacuum solutions derived in this section provide all the vacuum and Petrov type $D$ Kundt's spacetimes foliated by the non-expanding horizons.

\section{Acknowledgments}

We would like to thank Abhay Ashtekar, Jiri Bicak, Piotr Chrusciel, Sean Hayward, Ted Jacobson, Jorma Louko and Bernd Schmidt for discussions. This work was supported in part by the Polish Committee for Scientific Research (KBN) under grant numbers 2 P03B 073 24, 2 P03B 127 24, 2 P03B 130 24, the Albert Einstein Institute of the Max Planck Society and Batory fellowship. 


\section{Appendix A. A topological constraint}

From the geometrical point of view the definition of a non-expanding horizon may be generalized to an arbitrary compact 2-manifold $S$. Note that every non-orientable $S$ admits a double covering by the orientable one. Therefore, we can assume that $S$ is orientable and fix an orientation in $S$ the same way as in the case of $S$ diffeomorphic to the 2-sphere (see section 2). Then the definitions of the structures $(\hat{q}, \hat{\omega}, \hat{\mathcal{F}})$ introduced in section 2 still apply, as well as propositions 1 and 2. Let as consider that generalization in this section (only) to prove the following topological consequence of the constraints:

Theorem 4. Suppose $(M, g, F)$ is an electrovac foliated by non-expanding horizons in the meaning of the conditions (ii), (iii) of section 3 and the condition (i) replaced by the following assumption: $M=S \times \mathcal{I} \times \mathcal{I}$, where $S$ is a compact, connected, orientable 2-manifold and $\mathcal{I}$ is a manifold diffeomorphic with an interval. Then $S$ is either the torus or the sphere. In the first case, the only solution of the constraints (18) is

$$
\hat{\omega}=0, \quad \hat{\mathcal{F}}=0
$$

and a flat metric $\hat{q}$.

Proof. The trace part of $(18 a)$ reads

$$
\mathrm{d} \hat{\star} \hat{\omega}+K \hat{\epsilon}=\left(\hat{q}^{A B} \hat{\omega}_{A} \hat{\omega}_{B}+2 \kappa_{0}\left|\Phi_{1}\right|^{2}\right) \hat{\epsilon},
$$

where $\hat{\star}$ and $K:=\frac{1}{2} \hat{q}^{A B} R_{A B}$ are, respectively, the Hodge star and the Gaussian curvature of $(S, \hat{q})$, and $\hat{q}^{A B}$ is the two-dimensional inverse metric. The integral of the equation along $S$ and the Gauss-Bonnet theorem give the genus of $S$,

$$
2-2 \mathbf{g}=\int_{S}\left(\hat{q}^{A B} \hat{\omega}_{A} \hat{\omega}_{B}+2 \kappa_{0}\left|\Phi_{1}\right|^{2}\right) \hat{\epsilon} .
$$

It follows immediately that

$$
\mathbf{g} \leqslant 1 .
$$

In the case of $\mathbf{g}=1$, that is when $S$ is a torus, equation (60) implies that $\hat{\omega}$ and $\Phi_{1}$ are identically 0 . Then, a consequence of $(18 a)$ is that the metric $\hat{q}$ on $S$ is Ricci flat.

In conclusion the only compact, orientable 2-manifolds which admit a solution of the constraints (18) are the 2-sphere and the 2-torus. It is not justified, however, to refer to a non-expanding null 3-surface whose section is topologically a torus as a 'horizon', because a surface of those properties can be admitted even by a flat geometry, for example, by Minkowski divided by a discrete subgroup of translations.

\section{Appendix B. The vacuum constraint equations}

In this section we will restrict ourselves to solutions to the constraint equations (18) with $\Phi_{1}=0$. This assumption does not restrict us to vacuum spacetimes-null electromagnetic radiation is still allowed.

By contracting the traceless part of $(18 a)$ with $\hat{\omega}^{A} \hat{\omega}^{B}$ (where indices of $\hat{\omega}$ are raised using inverse metric $\hat{q}^{A B}$ on $S$ ), we obtain the following identity:

$$
\hat{q}^{A B} \hat{\omega}_{A} \hat{D}_{B}|\hat{\omega}|^{2}=|\hat{\omega}|^{2} \operatorname{div} \hat{\omega}+|\hat{\omega}|^{4},
$$

where

$$
|\hat{\omega}|^{2}=\hat{q}^{A B} \hat{\omega}_{A} \hat{\omega}_{B}, \quad \operatorname{div} \hat{\omega}=\hat{q}^{A B} \hat{D}_{A} \hat{\omega}_{B}
$$


From this equation and (59) it follows that the following equality holds for each real $\beta$ :

$$
\hat{q}^{A B} \hat{D}_{A}\left(|\hat{\omega}|^{2 \beta} \hat{\omega}_{B}\right)=(2 \beta+1)|\hat{\omega}|^{2(\beta+1)}-(\beta+1)|\hat{\omega}|^{2 \beta} K .
$$

That finally implies one-parameter family of integral identities

$$
\frac{2 \beta+1}{\beta+1} \int_{S}|\hat{\omega}|^{2(\beta+1)} \hat{\epsilon}=\int_{S} K|\hat{\omega}|^{2 \beta} \hat{\epsilon} .
$$

Suppose $\hat{\omega}$ has only a finite set of critical points (that is such that $|\hat{\omega}|=0$ ) which are isolated. Then equation (64) for $\beta=-\frac{1}{2}$ takes the form

$$
\hat{q}^{A B} \hat{D}_{A} \frac{\hat{\omega}_{B}}{|\hat{\omega}|}=-\frac{K}{2|\hat{\omega}|} .
$$

That equation can be integrated over $S$ avoiding the singularities by surrounding the critical points by small circles and passing to the limit (i.e. shrinking circles to critical points). It provides the special case of equation (65)

$$
\int_{S} \frac{K}{|\hat{\omega}|} \hat{\epsilon}=0 .
$$

This condition implies that $K$ must be negative on some open subset of $S$. The considerations can be summarized by the following

Theorem 5. There are no solutions to constraints (18) of the following properties:

(a) projected electromagnetic field tensor $\hat{\mathcal{F}}$ vanishes,

(b) rotation 1-form $\hat{\omega}$ vanishes only at a finite set of points,

(c) $S$ is a sphere with non-negative Gaussian curvature.

The Gauss-Bonnet theorem implies that $\hat{\omega} \neq 0$ on an open non-empty subset. On the other hand, the solution generated by projected data of the extremal Reissner-Nordström horizon (case $\alpha=1$ in (55)) is an example of solutions for Einstein-Maxwell equations with $\hat{\omega} \equiv 0$, which means that some arguments used above are no longer true for solutions describing fields other than null radiation.

\section{References}

[1] Wald R 1993 Black hole entropy is Noether charge Phys. Rev. D 48 3427-31 (Preprint gr-qc/9307038)

[2] Iyer V and Wald R 1994 Some properties of Noether charge and a proposal for dynamical black hole entropy Phys. Rev. D 50 846-64 (Preprint gr-qc/9403028)

[3] Wald R 2001 The thermodynamics of black holes Living Rev. Rel. 46 (Preprint gr-qc/9912119)

[4] Jacobson T, Kang G and Myers R 1994 On black hole entropy Phys. Rev. D 49 6587-98 (Preprint gr-qc/9312023)

[5] Ashtekar A, Beetle C, Dreyer O, Fairhurst S, Krishnan B, Lewandowski J and Wisniewski J 2000 Generic isolated horizons and their applications Phys. Rev. Lett. 85 3564-7 (Preprint gr-qc/0006006)

[6] Ashtekar A, Beetle C and Lewandowski J 2002 Geometry of generic isolated horizons Class. Quantum Grav. 19 1195-225 (Preprint gr-qc/0111067)

[7] Ashtekar A and Krishnan B 2002 Dynamical horizons: energy, angular momentum, fluxes and balance laws Phys. Rev. Lett. 89261101 (Preprint gr-qc/0207080)

[8] Hayward S 1994 General laws of black-hole dynamics Phys. Rev. D 49 6467-74 (Preprint gr-qc/9303006)

[9] Hayward S 1993 On the definition of averagely trapped surfaces Class. Quantum Grav. 10 L137-40 (Preprint gr-qc/9304042)

[10] Jezierski J, Kijowski J and Czuchry E 2000 Geometry of null-like surfaces in general relativity and its application to dynamics of gravitating matter Rep. Math. Phys. 46 399-418

[11] Racz I and Wald M 1992 Extensions of spacetimes with Killing horizons Class. Quantum Grav. $92643-56$ 
[12] Kramer D, Stephani H, MacCallum M and Herlt E 2003 Exact Solutions of the Einsteins Field Equations 2nd edn (Cambridge: Cambridge University Press)

[13] Lewandowski J and Pawlowski T 2003 Extremal isolated horizons: A local uniqueness theorem Class. Quantum Grav. 20 587-606 (Preprint gr-qc/0208032)

[14] Nakahara M 2003 Geometry, Topology and Physics 2nd edn (Bristol: Institute of Physics Publishing)

[15] Ashtekar A, Beetle C and Lewandowski J 2002 Mechanics of rotating isolated horizons Phys. Rev. D 6404401 (Preprint gr-qc/0103026) 